\title{
A contribuição de Frederico Lange de Morretes para a malacologia brasileira
}

\author{
Marcos de Vasconcellos Gernet ${ }^{1,4}$; Carlos Eduardo Belz ${ }^{1,2,5}$; Carlos João Birckolz ${ }^{1,6}$; \\ Luiz Ricardo Lopes de Simone ${ }^{2,7}$ \& Claudia Inês Parellada ${ }^{3}$ \\ ${ }^{1}$ Universidade Federal do Paraná (UFPR), Centro de Estudos do Mar (CEM), Laboratório de Ecologia Aplicada e Bioinvasões (LEBI0). \\ Pontal do Paraná, PR, Brasil. \\ 2 Universidade de São Paulo (USP), Museu de Zoologia (MZUSP). São Paulo, SP, Brasil. \\ 3 Museu Paranaense, Setor de Arqueologia. Curitiba, PR, Brasil. ORCID: http://orcid.org/0000-0002-1603-3144. E-mail: cparellada34@gmail.com \\ ${ }^{4}$ ORCID: http://orcid.org/0000-0001-5116-5719. E-mail:Imv.gernet@gmail.com \\ ${ }^{5}$ ORCID: http://orcid.org/0000-0002-2381-8185. E-mail: belzoceanos@gmail.com \\ ${ }^{6}$ ORCID: http://orcid.org/0000-0002-7896-1018. E-mail: carlosbirc@gmail.com \\ ${ }^{7}$ ORCID: http://orcid.org/0000-0002-1397-9823. E-mail: Irsimone@usp.br
}

\begin{abstract}
Frederico Lange de Morretes was born on May 5, 1892, in the municipality of Morretes, Paraná, Brazil. A renowned plastic artist, he was also an important malacologist, and his scientific production in the area made him a reference for Brazilian researchers. The main objective of this paper is to make a commented compilation of the malacological studies produced by him. He published 13 scientific papers in the area of malacology, eight of them related to the description of 25 new species, two new genera and three subgenera. He also wrote three institutional technical reports on activities carried out at the Museu Paulista and at the Museu Paranaense.
\end{abstract}

Key-Words. Lange de Morretes; Malacology; Scientific production.

\begin{abstract}
Resumo. Frederico Lange de Morretes nasceu em 5 de maio de 1892, no município de Morretes, litoral do Estado do Paraná, Brasil. Artista plástico renomado, foi também um importante malacólogo e sua produção científica na área o tornou um referencial para os pesquisadores brasileiros. 0 principal objetivo deste trabalho é a compilação comentada dos estudos malacológicos produzidos por Lange de Morretes. Ao todo, ele publicou 13 trabalhos científicos na área da malacologia, sendo oito deles relacionados à descrição de 25 novas espécies, dois novos gêneros e três subgêneros. Também escreveu três relatórios técnicos institucionais a respeito de atividades desenvolvidas no Museu Paulista e no Museu Paranaense.
\end{abstract}

Palavras-Chave. Lange de Morretes; Malacologia; Produção científica.

\section{INTRODUÇÃO}

Frederico Godofredo Lange nasceu em 5 de maio de 1892 no município de Morretes, litoral do Estado do Paraná, Brasil, e faleceu em 19 de janeiro de 1954, em Curitiba, Paraná. Entre os dois e os nove anos de idade, viveu na casa do Ypiranga, entre os quilômetros 71 e 72 da estrada de ferro Curitiba-Paranaguá, no meio da Serra do Mar. Este contato direto com a natureza na infância influenciou de forma definitiva sua carreira artística e científica (Salturi, 2007).

A família de Lange de Morretes possuía laços de amizade com o pintor norueguês Alfredo Andersen, um dos precursores da arte paranaense, que ministrou aulas para o menino Frederico desde os nove anos de idade e participou de sua formação como artista (Corrêa, 2014). A família apoiou seus estudos na Alemanha, entre 1910 e 1920, cursando, inicialmente, a Real Academia de Artes Gráficas de Leipzig (Konigliche Akademie für Graphische Künste und Buchgewerbe). Em 1914, foi admitido na Academia de Belas Artes de Munique (Akademie der Bildenden Künste München), concluindo a graduação em Pintura e Escultura em 1920. Durante sua formação também cursou várias disciplinas da área biológica (Salturi, 2009a).

Em Munique, acrescentou "de Morretes" ao nome, pois o reitor solicitou que os alunos com nomes iguais fossem ao cartório e inserissem ao sobrenome a cidade natal de cada um, assim não haveria confusões nas notas e na aplicação de advertências. Lange também era um sobrenome muito comum na Alemanha, por isso ele decidiu 
usar o nome artístico (Salturi, 2007, 2009a). O sobrenome composto Lange de Morretes aparece, em 1914, na carteira estudantil da Academia de Belas Artes de Munique, doada pela família ao Museu Paranaense, em 2017.

Os primeiros trabalhos referentes à malacologia foram um artigo sobre dois novos gastrópodes pulmonados do Brasil, publicado em novembro de 1937 e o relatório de atividades da seção de malacologia, apresentado em janeiro de 1937, relativo ao período em que foi assistente na Cátedra de Paleontologia do Museu Paulista, sob responsabilidade do vulcanólogo italiano, professor Barão Ottorino De Fiore di Cropani. Lange de Morretes foi convidado para atuar no Museu Paulista, em 1935, pelo entomólogo Frederico Lane, que trabalhava no Setor de Zoologia do mesmo museu (Salturi, 2007).

A mudança de Curitiba, com a família, para São Paulo, em 1936, foi amplamente relatada por Lange de Morretes em diferentes textos de periódicos, como Illustração Paranaense e Gazeta do Povo, entre outros. Nestas narrativas descreve sua trajetória na formação de alunos em artes plásticas, em escolas-atelier e redes escolares, na criação de entidades de classe, e no rompimento com o Interventor do Estado do Paraná, Manoel Ribas, devido a promulgação de lei estadual que taxava as obras de arte (Morretes, 1931/1943).

Questões políticas, porém, fizeram com que sua nomeação como Diretor do Museu Paranaense, em 1936, não fosse efetivada e, José Loureiro Fernandes, médico urologista, antropólogo e político assumisse o cargo. A mágoa desse fato, somado a saída de Lange de Morretes da Escola Normal de Curitiba, fez com que decidisse dedicar, com maior intensidade, à malacologia na Universidade de São Paulo.

A data de 1937, da primeira publicação, não significa que o interesse pela malacologia tenha começado naquele ano, pois as coletas de exemplares efetuados por Lange de Morretes, que resultariam em temas da maioria de seus estudos, remetem ao início da década de 1920, conforme análises de dados dos dois livros tombo da sua coleção particular, que se encontram depositados no laboratório de malacologia do Museu de Zoologia da Universidade de São Paulo (MZUSP).

Lange de Morretes periodicamente retornava ao Paraná, especialmente à Curitiba a partir de 1941, para participar de eventos, como exposições coletivas e realizar coletas. Em 1948, participou da fundação da Escola de Música e Belas Artes do Paraná (EMBAP), atuando como professor de anatomia e fisiologia (Salturi, 2007). Em 1950, tornou-se pesquisador do Museu Paranaense, que estava, naquela época, sob mandato da Universidade do Paraná (atual Universidade Federal do Paraná - UFPR). Em 1953, recebeu uma bolsa do Conselho Nacional de Pesquisas (CNPq) para realizar levantamento malacológico no litoral sul-sudeste do Brasil (Salturi, 2009a). Durante sua vida, teve grande preocupação com a questão ambiental, principalmente com o desmatamento que ocorria no Estado do Paraná à época (Morretes, 1953b).

Lange de Morretes é mais conhecido como renomado artista plástico: pintor, desenhista, gravador e escultor, que transitou entre o impressionismo, o realismo e o modernismo, sendo um dos articuladores do Movimento Paranista (Salturi, 2009b); muitos dados novos sobre sua trajetória artística e acadêmica estão sendo revelados devido à descoberta, em 2017, de textos inéditos manuscritos e datilografados.

Sua atuação na malacologia, apesar de toda a sua produção científica na área, que o tornou um referencial para pesquisadores brasileiros, foi pouco estudada. Para diminuir a lacuna que existe referente à sua vida científica, este trabalho buscou compilar os textos científicos e técnicos de Lange de Morretes na área da malacologia, com comentários sobre os mesmos e sobre as espécies estudadas por ele.

\section{TRABALHOS CIENTÍFICOS MALACOLÓGICOS DE LANGE DE MORRETES}

Ao todo Frederico Lange de Morretes publicou 13 trabalhos científicos na área da malacologia, sendo oito deles relacionados à descrição de 25 novas espécies, incluindo dois novos gêneros e três subgêneros. Dentre suas produções científicas, a mais importante foi "Ensaio de Catálogo dos Moluscos do Brasil", publicada em 1949 e, que será mais amplamente discutida na sequência. Também comentaremos sobre os dois relatórios de atividades desenvolvidas em 1936 e 1937, enviados ao diretor do Museu Paulista na época, e sobre o relatório de atividades institucionais desempenhadas no Museu Paranaense, em 1953.

O primeiro trabalho analisado foi o "Relatório da Secção de Malacologia do Anno de 1936" (Simone, 2003b), enviado no dia 2 de janeiro de 1937, com intuito de prestar contas sobre as atividades desempenhadas na coleção de invertebrados (Malacologia) do Museu Paulista ao longo do ano de 1936.

Nesse relatório, percebe-se por parte do autor que "o principal obstáculo, nos trabalhos empreendidos, é a falta de literatura scientifica moderna com respeito ao ramo" no museu. Dentre as atividades relatadas estão a conservação das coleções (desinfecção de armários, confecção de novas etiquetas, novo acondicionamento dos exemplares, calibragem do álcool da coleção em meio líquido e organização sistemática); tombamento de materiais, chamados no texto de legados; excursão ao litoral paulista para coleta de material biológico que ocorreu do dia 15 de julho a 9 de setembro de 1936 e estudo específico de cada grupo de invertebrados, contando com a colaboração de especialistas nas diferentes áreas.

Em 17 de novembro de 1937, publica o artigo "Dois novos Gasteropodos Pulmonados do Brasil" (Simone, 2003a), na Revista do Museu Paulista, Tomo XXIII (Fig. 1A), descrevendo duas novas espécies de gastrópodes terrestres.

A primeira foi Megalobulimus toriii Morretes, 1937, coletada por ele em abril de 1937 à profundidade de um metro, no sambaqui do Morro do Bernardes, Jupuvura, município de Iguape, no Estado de São Paulo, durante sua excursão a este sítio arqueológico acompanhando o 
arqueólogo e paleontólogo japonês Ryuzo Torii. O nome da espécie é uma homenagem àquele pesquisador.

A outra espécie descrita por Lange de Morretes naquele trabalho foi Coniclus jaussaudi = Mirinaba jaussaudi (Morretes, 1937), coletada por Jorge Santos em julho de 1934, em Morungava, no município de Sengés, no Estado do Paraná. O nome da espécie é uma homenagem ao Dr. Paul Jaussaud, que entregou a Lange o exemplar em julho do mesmo ano.

Seu próximo trabalho foi o "Relatório da Secção de Malacologia do Anno de 1937" (Simone, 2003b) apresentado em 2 de janeiro de 1938. Desta vez o texto começa com menção ao nome do diretor do Museu Paulista e historiador, Dr. Affonso d'Escragnolle Taunay. Naquele trabalho pode-se observar uma prestação de contas relativa às atividades desempenhadas na curadoria da coleção de invertebrados, principalmente na de moluscos, como a continuação nos trabalhos de conservação dos materiais; determinação dos espécimes coletados na excursão ao litoral paulista por Barão de Fiore di Cropani e também troca de materiais com outras instituições não mencionadas.

Um dos pontos importantes desse relatório é a menção à passagem pelo Museu Paulista, do pesquisador Japonês Ryuzo Torii, o qual, como mencionado acima, foi acompanhado por Lange de Morretes em pesquisas ao sambaqui do Morro do Bernardes, onde foi coletado farto material malacológico.

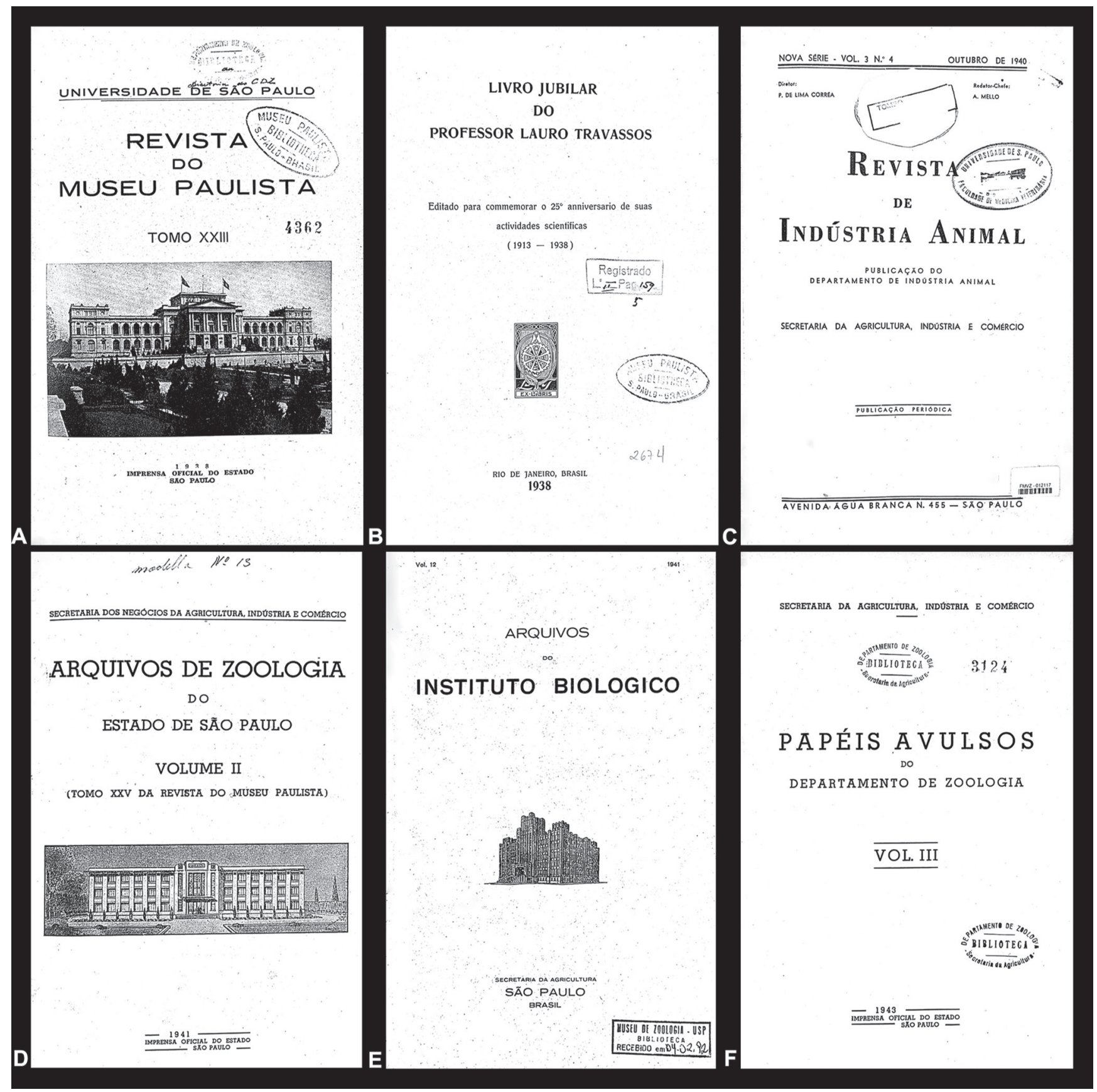

Figura 1. Capas originais dos periódicos onde os trabalhos de Lange de Morretes foram publicados, por ordem cronológica. (A) = Revista do Museu Paulista (Morretes, 1937a); (B) = Livro Jubilar do Professor Lauro Travassos (Morretes, 1938a); (C) = Revista de Indústria Animal (Morretes, 1940a); (D) = Arquivos de Zoologia do Estado de São Paulo (Morretes, 1940b, c); (E) = Arquivos do Instituto Biológico (Morretes, 1941); (F) = Papéis Avulsos do Departamento de Zoologia (Morretes, 1943). 
Nesse relatório, Lange comenta sobre a importante aquisição por parte da biblioteca do museu da obra Handbuch der Systematischen Weichtierkunde (Manual de Malacologia Sistemática), do professor Johannes Thiele. Mesmo assim deixa clara a necessidade de outras aquisições literárias: "Seria de grande utilidade completar o "Manual of Conchology", de Pilsbry, indispensável ao estudo dos Molluscos Pulmonados, e do qual o museu já possui 17 volumes".

Também em 1938, foi publicado como contribuição ao Livro Jubilar em homenagem ao eminente zoólogo Lauro Travassos (Fig. 1B) o artigo "Duas espécies novas de molluscos marinhos do Brasil" (Morretes, 1938a). Nessa publicação encontra-se a descrição do gastrópode marinho pertencente à família Olividae, Agaronia travassosi Morretes, 1938, coletado durante uma excursão de dois meses para coleta de material malacológico, por Lange de Morretes, na Praia do Gato, Baía dos Castelhanos, Ilha de São Sebastião, Estado de São Paulo, em julho de 1936. Esta espécie foi uma homenagem a Lauro Travassos e, nesse trabalho, pela primeira vez Lange de Morretes representa o ambiente em que foram coletados os exemplares através de uma ilustração em aquarela, fortalecendo a relação "artista-cientista".

A outra espécie descrita, também pertencente à família Olividae, foi Agaronia lanei Morretes, 1938, coletada em Guaratuba, no Estado do Paraná, por Lange de Morretes, em 1927. No entanto, de acordo com Rios (2009), posteriormente constatou-se que este táxon é um sinônimo de $A$. travassosi.

Em outubro de 1940, na Revista de Indústria Animal, Vol. 3, N. 4 (Fig. 1C), foi publicado o artigo "Algumas palavras sôbre novas ocorrências e maior distribuição de moluscos na costa do Brasil" (Morretes, 1940a). Naquele artigo o autor discute a necessidade de pesquisas mais aprofundadas para demonstrar a distribuição geográfica mais ampla de determinadas espécies de moluscos tanto para o norte (fauna das Antilhas) quanto para o sul (fauna Patagônica), pois as pesquisas malacológicas se tornaram raras após a morte do zoólogo Herman von Ihering, diretor do Museu Paulista (1894-1915). O autor analisou vários exemplares de moluscos coletados por Barão de Fiore di Cropani em excursões ao litoral paulista, constatando novas ocorrências para a costa de São Paulo e indivíduos ainda não encontrados no Brasil.

Outro assunto importante abordado naquele trabaIho foi a constatação da ocorrência de grande quantidade do gastrópode Helix aspersa Müller, 1774 = Cornu aspersum, em terrenos baldios na Rua Cabral, às margens do Rio Ivo, no município de Curitiba, no outono de 1931. Lange de Morretes ao longo do trabalho questiona de que forma este animal teria aparecido e se propagado pela cidade. Era um tema a ser investigado. Em conversa com o francês sr. Chautard, o autor descobre que este em uma viagem para o Rio de Janeiro, no final do séc. XIX, traz, a pedido de seu sócio, um lote de caracóis vindos da França para utilizar como remédio contra "doenças do peito". No entanto, como o consumo foi considerado não agradável, os animais foram soltos no quintal de sua casa na Praça Tiradentes, tendo, posteriormente, se espalhado por Curitiba.

No dia 22 de dezembro de 1940, foi publicado nos Arquivos de Zoologia do Estado de São Paulo, Vol. 2, Tomo XXV (Fig. 1D), o artigo intitulado "Novos moluscos marinhos do Brasil" (Morretes, 1940b). Nesse trabalho, Lange de Morretes descreveu a espécie Typhis melloleitaoi Morretes, 1940, pertencente à família Muricidae, coletada por Barão de Fiore di Cropani, na praia de Mococa, Caraguatatuba, Estado de São Paulo, em 1940. O nome foi uma homenagem ao zoólogo, fundador da Aracnologia na América do Sul, Cândido Firmino de Mello-Leitão. Segundo Rosenberg (2009), o táxon é um sinônimo de Typhis expansus Sowerby, 1874.

Persicula dalii teve sua descrição feita de forma diferenciada. Analisando um exemplar coletado por Barão de Fiore di Cropani, em Mococa, Caraguatatuba, Estado de São Paulo, o autor fez a análise comparativa de indivíduos existentes na coleção do Museu Paulista, tendo encontrado um exemplar bastante semelhante ao coletado, cuja etiqueta continha as seguintes anotações: "Persicula dalii ih." e a observação de que o material havia sido analisado por Dall, em 1904, e coletado pelo sr. Bittencourt, em 1900, em Bertioga, Santos. A letra, segundo Morretes (1940b), era indubitavelmente de von Ihering, no entanto revendo toda a literatura referente a então família Marginellidae não encontrou nenhuma referência à espécie catalogada, motivo pelo qual resolveu descrevê-la reutilizando este mesmo nome. De acordo com Rosenberg (2009), este táxon é um sinônimo de Hesperato maugeriae (Gray, 1832).

Outra espécie descrita também naquele trabalho foi Leda schuberti Morretes, 1940, da família Ledidae = Nuculanidae, coletada durante dragagem no Porto de Paranaguá, no Estado do Paraná, em 10 de agosto de 1934, por Lange de Morretes, e cujo nome foi uma homenagem ao seu irmão, o engenheiro Carlos Augusto Schubert, falecido em 1940. Esse táxon é um sinônimo de Adrana patagonica (d'Orbigny, 1846), de acordo com Rosenberg (2009).

No dia 27 de dezembro de 1940, foi publicado também nos Arquivos de Zoologia do Estado de São Paulo, Vol. 2, Tomo XXV (Fig. 1D) o artigo "Um novo Gasterópodo Pulmonado do Brasil" (Morretes, 1940c), no qual há a descrição da espécie Entodina gionensis Morretes, 1940, da família Systrophiidae (atualmente Scolodontidae), coletada pelo Barão de Fiore di Cropani, em 1939, no sambaqui de Vamiranga, município de Iguape, Estado de São Paulo. Esta espécie recebeu seu nome em homenagem ao secretário de Educação e da Saúde Pública do Estado de São Paulo, Dr. Álvaro Guião.

Em outubro de 1941, na Revista Arquivos do Instituto Biológico, Vol. 12 (Fig. 1E), foi publicado o artigo sobre o bivalve dulcícola da família Mycetopodidae, "Rochanaia gutmansi, nov. gen. n. sp., novo Lamelibrânquio do Brasil" (Morretes, 1941), apresentado para o volume jubilar do professor Rocha Lima, no qual Lange de Morretes descreve não apenas uma nova espécie, mas também um novo gênero. O exemplar foi coletado no Rio Nioaque, Lugar Cedro, município de Nioaque, Estado de Mato Grosso 
(atualmente Mato Grosso do Sul), em 13 de abril de 1939 por Lange de Morretes, auxiliado em suas coletas pelo agrimensor Carlos Dobes. De acordo com Simone (2006), o gênero Rochanaia Morretes, 1941 é sinônimo de Bartlettia A. Adams, 1866, e a espécie R. gutmansi Morretes, 1941 é sinônima de B. stephanensis (Moricand, 1856).

O nome do novo gênero foi uma homenagem ao ilustre médico sanitarista e patologista, um dos fundadores do Instituto Oswaldo Cruz, Dr. Henrique da Rocha Lima, e o nome da espécie foi uma homenagem ao geólogo, descobridor da famosa Rocha Moutonée, Dr. Marger Gutmans, que foi o coletor do primeiro exemplar, julgando ser um fóssil.

No dia 11 de junho de 1943, nos Papéis Avulsos do Departamento de Zoologia, Vol. 3, N. 7 (Fig. 1F), foi publicado o artigo "Contribuição ao Estudo da Fauna Brasileira de Moluscos: Resultados de uma pequena coleção de moluscos obtida pela excursão científica realizada pelo Instituto Osvaldo Cruz em outubro de 1938" (Morretes, 1943). Naquele trabalho, Lange de Morretes analisa o material coletado na expedição científica pelo Dr. Frederico Lane, assistente do Departamento de Zoologia da Secretaria de Agricultura de São Paulo, e também pelo Dr. Romeu Cuocolo, do Instituto Biológico de São Paulo. No artigo foi realizada a identificação do material encontrado e uma avaliação sistemática de todas as 19 espécies (gastrópodes prosabrânquios e pulmonados, e bivalves límnicos) coletadas durante a expedição científica.

Em dezembro de 1949 foi publicada nos Arquivos do Museu Paranaense, Vol. 7 (Fig. 2A), a mais importante obra de Frederico Lange de Morretes, intitulada "Ensaio de Catálogo dos Moluscos do Brasil" (Morretes, 1949), que, segundo o próprio autor, "representava o primeiro arrolamento de moluscos do Brasil, e que embora longe de ser completo, era todavia um passo avante na catalogação da nossa fauna e que caso fosse útil aos interessados teria preenchido a sua finalidade". Esse trabalho continha 211 páginas de listagem sobre taxonomia e distribuição da fauna malacológica brasileira, não só em território nacional, mas também de suas ocorrências em outros países. No artigo foram apresentadas cerca de 1.500 espécies de moluscos, pertencentes às classes Polyplacophora, Bivalvia, Scaphopoda, Gastropoda e Cephalopoda.

Em 1952 foi publicado nos Arquivos de Zoologia do Estado de São Paulo, Vol. 8 (Fig. 2B), o trabalho "Novas Espécies Brasileiras da Família Strophocheilidae" (Morretes, 1952), cujo texto aborda a escassez de literatura referente aos estudos de biologia e anatomia da família Strophocheilidae. De acordo com o autor, baseando-se em caracteres conquiliológicos, ele procurou sistematizar o referido grupo, revisando o trabalho de Bequaert (1948) (o qual também abordou os Strophocheilidae), reunindo a este dois gêneros restritos ao Brasil: Gonyostomus Beck, 1837 e Anthinus Albers, 1850. Também elevou à categoria de gênero, vários subgêneros que apresentavam caracteres suficientes para isto, criando também pelo mesmo motivo novos subgêneros, como é o caso de Mirinaba, Metara e Phaiopharus. Apresentou também nesse trabalho um gênero novo denominado Psiloicus, atualmente sinônimo de Megalobulimus K. Miller, 1878. Nesse artigo também foi realizada a descrição de 13 novas espécies, todas ainda válidas, exceto Psiloicus bereniceae, que é considerada sinônima de Megalobulimus intertextus (Pilsbry, 1895), de acordo com Salvador et al. (2018b).

No mês de dezembro de 1953, foi publicado nos Arquivos do Museu Paranaense, Vol. 10, $1^{\text {a }}$ parte (Fig. 2C), o trabalho "Adenda e Corrigenda ao Ensaio de Catálogo dos Molusculos do Brasil" (Morretes, 1953a), tratando das correções e comentários sobre o seu trabalho "Ensaio de Catálogo dos Moluscos do Brasil", com acréscimo de literatura. Estas correções incluíam substituições de famílias, subfamílias, gêneros, subgêneros e espécies. Também foi observado o acréscimo de espécies e correções de localidades.

Ainda, de 19 de dezembro de 1953, existe um relatório de atividades no Museu Paranaense, de Lange de Morretes, cuja síntese, com quatro páginas, foi inserida no Livro de Relatórios Técnicos 1950-1969, devendo ser observado que não constam relatórios de alguns anos, como 1952. Morretes (1953c) relatou que realizou pesquisas no litoral do Estado do Paraná, em Matinhos, de 2 a 27 de junho de 1953, em Pontal do Sul, de 27 de julho a 25 de agosto, e na Ilha do Mel, de 10 a 28 de outubro. Comentou na página 84 que: "O Pontal do Sul, devido a sua situação, ofereceu ótimas condições para coleta de Moluscos, tanto de Opistobrânquios do Mar de Dentro quanto de Lamelibrânquios provindos de bancos fronteiriços". Relacionou as espécies novas de moluscos, que teriam artigos publicados no ano seguinte, e solicitou ao reitor da Universidade do Paraná a prorrogação do contrato, por mais um ano, para poder ampliar as pesquisas malacológicas na baía de Paranaguá.

Em dezembro de 1954 foi publicada nos Arquivos do Museu Paranaense, Vol. 10, 2a parte (Fig. 2D), o trabalho "Dois novos Moluscos do Brasil" (Morretes, 1954a), cujo texto tratou da descrição de duas novas espécies. A primeira espécie descrita, Adelopoma brasiliense, pertenceu à família Cyclophoridae, atualmente alocado na família Diplommarinidae, foi coletada pelo autor em duas localidades distintas (Sambaqui de Jupuvura, no município de Iguape, no Estado de São Paulo, e também em uma área de roçado, na região de Cerro Azul, no Estado do Paraná). Em ambas as localidades, os exemplares de Adelopoma brasiliense foram encontrados no interior de conchas vazias de espécies de Strophocheilidae.

A segunda espécie, Caecum (Elephantulum) berthae foi coletada na localidade chamada de Saco da Virgem, Barra Seca, na Praia de Perequê-Açú, em Ubatuba, litoral do Estado de São Paulo, pela esposa do autor, Bertha Lange de Morretes, em maio de 1950, a quem este dedicou o nome da espécie. De acordo com Rosenberg (2009), este táxon é sinônimo de Caecum plicatum Carpenter, 1858.

Também no ano de 1954, no mesmo volume 10 , $2^{a}$ parte dos Arquivos do Museu Paranaense (Fig. 2D), Frederico Lange de Morretes publicou a descrição de 


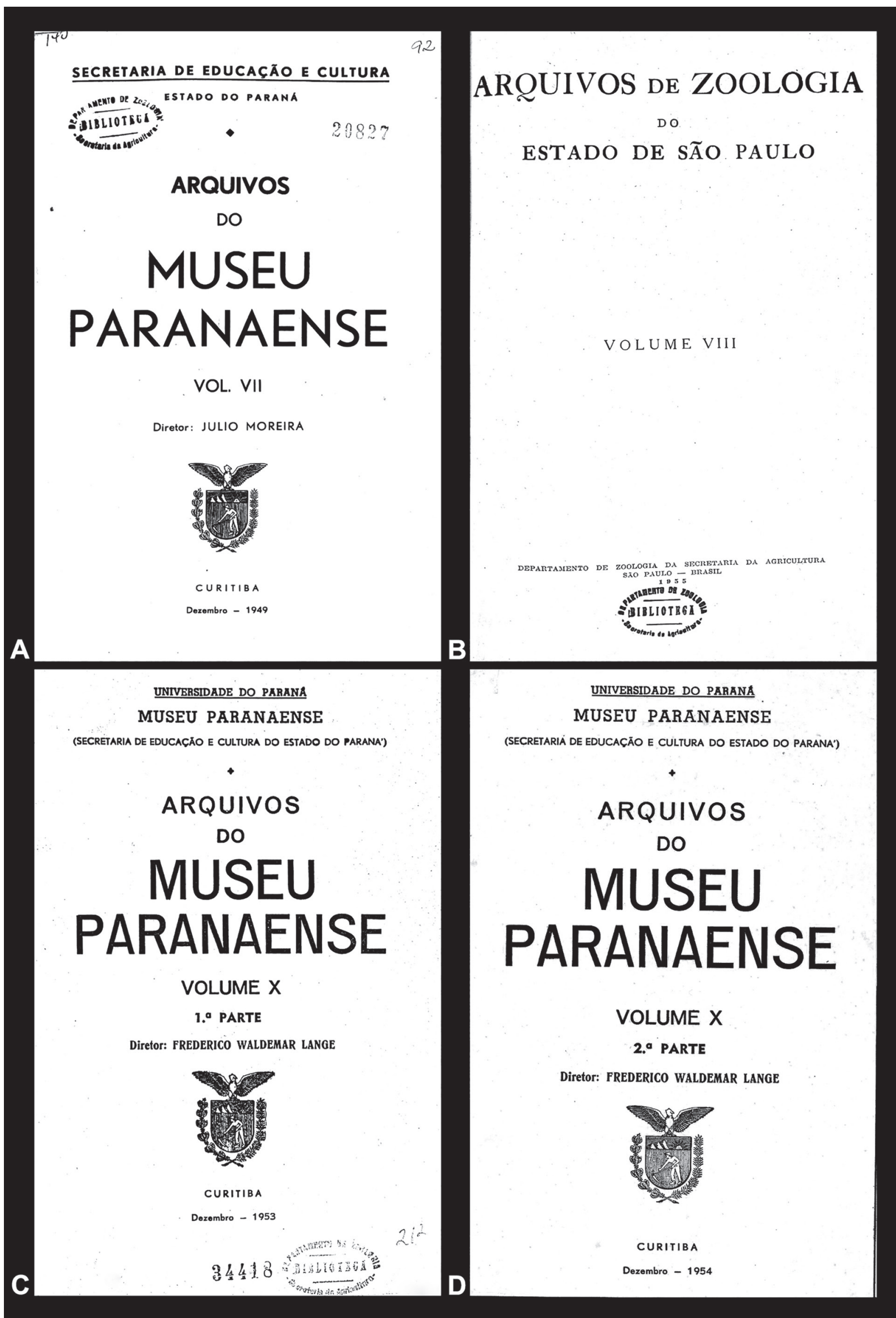

Figura 2. Capas originais dos periódicos onde os trabalhos de Lange de Morretes foram publicados, por ordem cronológica. (A) = Arquivos do Museu Paranaense (Morretes, 1949); (B) = Arquivos de Zoologia do Estado de São Paulo (Morretes, 1952); (C) = Arquivos do Museu Paranaense (Morretes, 1953a); (D) = Arquivos do Museu Paranaense (Morretes, 1954a, b, c). 
mais uma espécie nova, com o trabalho intitulado "Nova Thais do Brasil" (Morretes, 1954b). Naquele artigo ele descreveu Thais (Thaisella) mariae (hoje em Thaisella), cujo holótipo foi coletado pelo próprio autor em Guaratuba, no litoral do Estado do Paraná, em 1927. Alguns parátipos foram coletados também pelo autor na localidade de Itapema, no município de Antonina (10 de julho de 1933) e em Guaraqueçaba (21 de junho de 1934), ambas no litoral do Paraná. O nome da espécie é uma homenagem à Maria Aparecida de Faria Cardoso, que foi sua auxiliar no Museu Paulista.

Mais uma vez, no volume $10,2^{\text {a }}$ parte dos Arquivos do Museu Paranaense do ano de 1954 (Fig. 2D), foi publicado o trabalho "Sôbre Megalobulimus paranaguensis Pilsbry \& lering [sic]" (Morretes, 1954c), no qual Lange de Morretes faz considerações sobre esta espécie descrita por Pilsbry e Ihering, em 1900, e cuja localidade-tipo é o município de Paranaguá, no litoral do Estado do Paraná. Seus comentários incluíram a distribuição geográfica da espécie que vai desde Garuva, Santa Catarina, até Peruíbe, Estado de São Paulo. Foi encontrada pelo autor na planície costeira do litoral paranaense e nos contrafortes da Serra do Mar (São João da Graciosa e Porto de Cima). Também foram coletados exemplares na Ilha do Mel e na Ilha das Peças. Naquele trabalho, Lange de Morretes faz uma observação com relação ao não aproveitamento desta espécie na culinária local, uma vez que é uma iguaria altamente proteica e com importância medicinal, através do uso de seu muco para cicatrização de feridas.

\section{ESPÉCIES VÁLIDAS DESCRITAS POR LANGE DE MORRETES}

Em seus trabalhos científicos Lange de Morretes descreveu 25 espécies, sendo que atualmente 18 destas são válidas. Abaixo são apresentadas informações sobre taxonomia e distribuição geográfica conhecida das espécies válidas. A classificação sistemática das espécies segue Bouchet \& Rocroi (2005).

\section{Gastropoda \\ Caenogastropoda \\ Superfamília Cyclophoroidea \\ Família Diplommatinidae \\ Gênero Adelopoma Döering, 1884 \\ Adelopoma brasiliense Morretes, 1954}

Fig. 3A

Adelopoma brasiliense Morretes, 1954a: 331 (estampa 24, figs. 1-8); Salgado \& Coelho, 2003: 153; Simone, 2006: 46 (fig. 56).

Distribuição: Iguape, Estado de São Paulo; Cerro Azul, Estado do Paraná; Vidal Ramos, Estado de Santa Catarina (Simone, 2006; Agudo-Padrón et al., 2014).

\section{Neogastropoda \\ Superfamília Muricoidea \\ Família Muricidae \\ Gênero Thaisella Clench, 1947 \\ Thaisella mariae (Morretes, 1954)}

Fig. 3B

Thais (Thaisella) mariae Morretes, 1954b: 339 (estampas 25-26).

Thais haemastoma Rios, 1970: 82.

Thais haemastoma floridana Rios, 1975: 91 (estampa 26, fig. 376).

Thais mariae Rios, 1985: 91 (estampa 32, fig. 399); Marini, 1988: 311 (figs. 1-12); Rios, 1994: 117 (estampa 38, fig. 494); Rios, 2009: 222 (fig. 551).

Thaisella mariae Claremont et al., 2013: 94; Simone, 2017: 111, 119.

Distribuição: Ilha Itaparica, no Estado da Bahia, até o litoral do Estado do Paraná (Rios, 2009).

Comentários: O material-tipo utilizado na descrição da espécie está extraviado (Marini, 1988).

\section{Superfamília Olivoidea Família Olividae \\ Gênero Agaronia Gray, 1839 \\ Agaronia travassosi Morretes, 1938}

Fig. 3C

Agaronia travassosi Morretes, 1938a: 330 (estampa 1, figs. 2-5); Morretes, 1949: 101; Rios, 1970: 102 (estampa 32, fig. 1); Rios, 1985: 111 (estampa 38, fig. 490); Rios, 1994: 146 (estampa 47, fig. 636); Rios, 2009: 278 (fig. 695).

Agaronia lanei Morretes, 1938a: 330 (estampa 1, figs. 6-7); Morretes, 1949: 101.

Agaronia langei Zanardini, 1960: 1 (figs. 1-2).

Distribuição: Litorais do Estado do Espírito Santo até Santa Catarina (Rios, 2009).

\section{Stylommatophora Superfamília Acavoidea Família Strophocheilidae Gênero Megalobulimus K. Miller, 1878 Megalobulimus arapotiensis Morretes, 1952} Fig. 3D

Megalobulimus (Megalobulimus) arapotiensis Morretes, 1952: 118 (estampas 1-2, fig. 8); Morretes, 1953a: 66.

Megalobulimus arapotiensis Salgado \& Coelho, 2003: 157; Simone, 2006: 207 (fig. 782).

Distribuição: Fazenda Rio do Peixe, Arapoti, Estado do Paraná (Morretes, 1952; Simone, 2006). 
Comentários: A localidade-tipo desta espécie é Fazenda Rio do Peixe, em Arapoti (antiga Cachoeirinha), no município de Jaguariaíva (atualmente Arapoti), no Estado do Paraná. Foi coletado um único exemplar morto, por Felipe Tod, em junho de 1935.

\section{Megalobulimus bertae Morretes, 1952}

Fig. 3E

Megalobulimus (Phaiopharus) bertae Morretes, 1952: 117

(estampas 3-4, fig. 5); Morretes, 1953a: 65.

Megalobulimus bertae Salgado \& Coelho, 2003: 157;

Simone, 2006: 207 (fig. 784).
Distribuição: Viçosa, Estado de Minas Gerais; Castelo, Estado do Espírito Santo (Oliveira \& Almeida, 1999; Simone, 2006).

Comentários: Coletados sete exemplares vivos em Viçosa, no Estado de Minas Gerais, pela filha do autor, Dra. Berta Lange de Morretes, em julho de 1951, à quem ele dedicou o nome da espécie.

\section{Megalobulimus cardosoi (Morretes, 1952)}

Fig. 3F

Strophocheilus (Megalobulimus) cardosoi Morretes, 1952: 119 (estampas 3-4, fig. 3); Morretes, 1953a: 67.

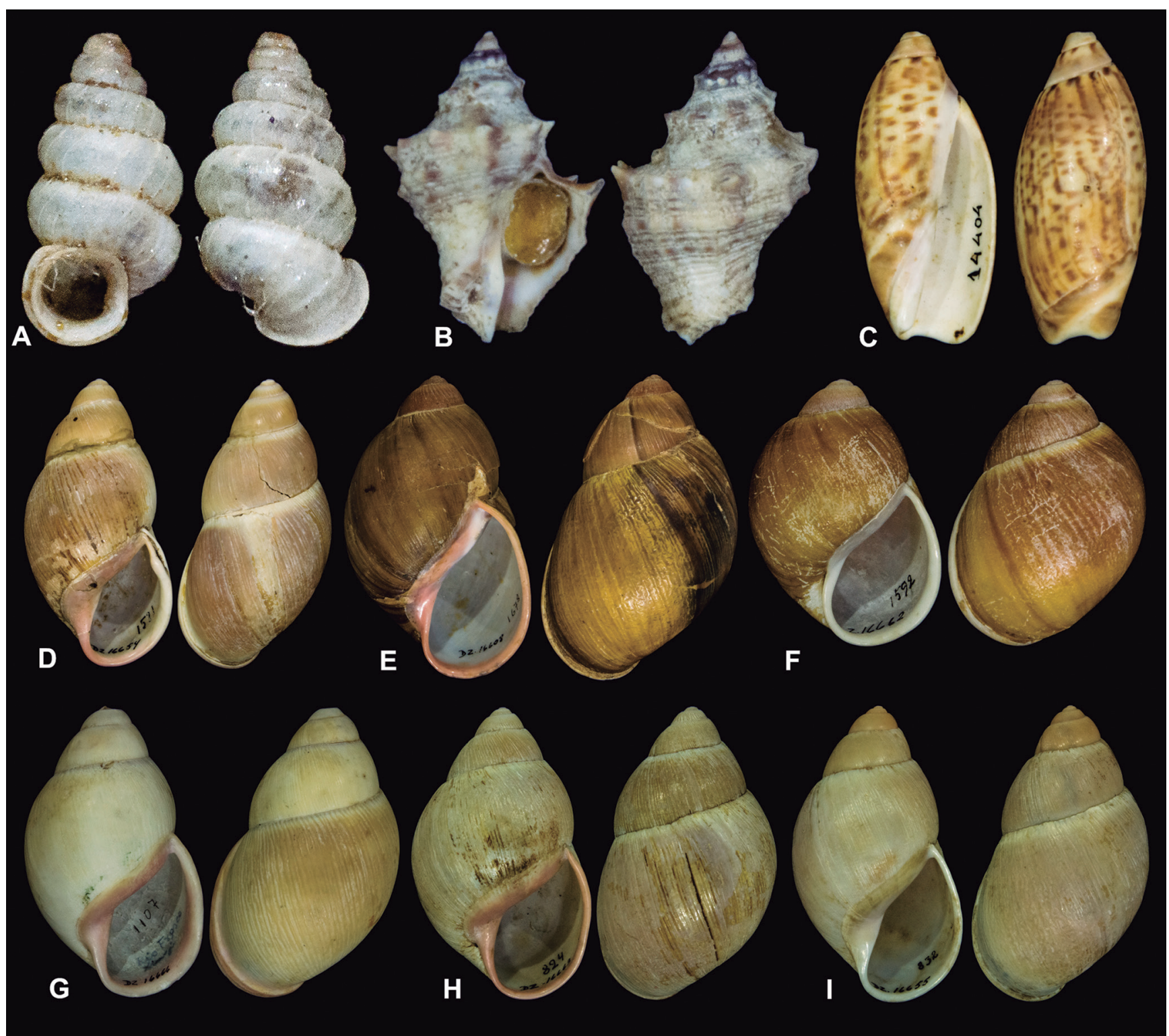

Figura 3. Espécies descritas por Lange de Morretes. H: Altura da concha. (A) = Adelopoma brasiliense, MZUSP 16863, Coleção Lange de Morretes, Parátipo, H=3 mm; (B) = Thaisella mariae, MZUSP 105293, Coleção Jorge Vaz, H = 27 mm; (C) = Agaronia travassosi, MZUSP 14104, Coleção Lange de Morretes, Holótipo, H = 44,6 mm; (D) = Megalobulimus arapotiensis, MZUSP 16654, Coleção Lange de Morretes, Holótipo, H= 85 mm; (E) = Megalobulimus bertae, MZUSP 16608, Coleção Lange de Morretes, Holótipo, H = 108 mm; (F) = Megalobulimus cardosoi, MZUSP 16662, Coleção Lange de Morretes, Holótipo, H=57 mm; (G) = Megalobulimus leonardosi, MZUSP 16666, Coleção Lange de Morretes, Holótipo, H=101,7 mm; (H)= Megalobulimus nodai, MZUSP 16663, Coleção Lange de Morretes, Holótipo, H=81,2 mm); (I) = Megalobulimus pintoi, MZUSP 16655, Coleção Lange de Morretes, Holótipo, H =84,2 mm. 
Megalobulimus cardosoi Salgado \& Coelho, 2003: 157; Simone, 2006: 208 (fig. 788); Salvador et al., 2018a: 119 (figs. 12A-B).

Distribuição: Murici e Reserva Biológica Serra Talhada, Estado de Alagoas (Salvador et al., 2018a).

Comentários: Foram coletados cinco exemplares no município de Murici, no Estado de Alagoas, pelo sr. Paulo de Sá Cardoso, a quem o autor dedicou o nome da espécie.

\section{Megalobulimus leonardosi (Morretes, 1952) Fig. 3G}

Psiloicus leonardosi Morretes, 1952: 125 (estampas 3-4, fig. 1); Morretes, 1953a: 69.

Megalobulimus leonardosi Salgado \& Coelho, 2003: 158; Simone, 2006: 213 (fig. 811).

Distribuição: Margens do Rio Fresco, afluente do Rio Xingu, Estado do Pará (Simone, 2006).

Comentários: Encontrado um único exemplar às margens do Rio Fresco, afluente do Rio Xingu, no Estado do Pará, pelo geólogo e engenheiro Dr. Othon Henry Leonardos, a quem o autor dedicou o nome da espécie.

\section{Megalobulimus nodai Morretes, 1952} Fig. $3 \mathrm{H}$

Megalobulimus (Megalobulimus) nodai Morretes, 1952: 120 (estampas 3-4, fig. 2); Morretes, 1953a: 67. Megalobulimus nodai Salgado \& Coelho, 2003: 158; Simone, 2006: 214 (fig. 817).

Distribuição: Margens do Rio Nhanpecein, afluente do rio Piquiri, Estado do Paraná (Simone, 2006).

Comentários: Coletado um único exemplar às margens do Rio Nhanpecein, afluente do Piquiri, no Estado do Paraná, por Sadamu Noda, em julho de 1935, a quem o autor dedicou o nome da espécie.

\section{Megalobulimus pintoi Morretes, 1952}

Fig. 31

Megalobulimus (Megalobulimus) pintoi Morretes, 1952: 121 (estampas 1-2, fig. 7); Morretes, 1953a: 68.

Megalobulimus pintoi Salgado \& Coelho, 2003: 158: 333; Simone, 2006: 217 (fig. 825).

Distribuição: Cuiabá, Estado do Mato Grosso (Simone, 2006).

Comentários: Coletado um único exemplar, em Cuiabá, no Estado do Mato Grosso, pelo ornitólogo e diretor do
Departamento de Zoologia, Dr. Olivério Mário de Oliveira Pinto, a quem o autor dedicou o nome da espécie.

\section{Megalobulimus rolandianus Morretes, 1952}

Fig. 4A

Megalobulimus (Megalobulimus) rolandianus Morretes, 1952: 122 (estampas 1-2, fig. 3); Morretes, 1953a: 68.

Megalobulimus rolandianus Salgado \& Coelho, 2003: 158; Simone, 2006: 219 (fig. 830).

Distribuição: Rolândia, Londrina, Estado do Paraná (Simone, 2006).

Comentários: Foram coletados quatro indivíduos, em Rolândia, no norte do Estado do Paraná, pela srta. Gertrud Altmann, dos quais foi selecionado o holótipo, e também um exemplar coletado em Londrina, Paraná, pelo sr. Dario Bastos, selecionado como parátipo.

\section{Megalobulimus toriii Morretes, 1937}

Fig. 4B

Megalobulimus toriii Morretes, 1937a: 301 (estampa 1, figs. 1-5); Salgado \& Coelho, 2003: 157; Simone, 2006: 221 (fig. 835).

Strophocheilus yporanganus toriii Bequaert, 1948: 141 (estampa 25, fig. 4).

Strophocheilus toriii Morretes, 1949: 142.

Megalobulimus (Phaiopharus) toriii Morretes, 1952: 113; Morretes, 1953a: 66.

Distribuição: Sambaqui do Morro do Bernardo, Jupuruva, Iguape, Estado de São Paulo; Sambaqui do Linguado, Estado de Santa Catarina (Simone, 2006).

\section{Megalobulimus wohlersi Morretes, 1952}

Fig. 4C

Megalobulimus (Megalobulimus) wohlersi Morretes, 1952: 123 (estampas 3-4, fig. 4); Morretes, 1953a: 68.

Megalobulimus wohlersi Salgado \& Coelho, 2003: 159; Simone, 2006: 221 (fig. 839); Salvador et al., 2018b: 82 (figs. 69-70).

Distribuição: Corumbá de Goiás, Estado de Goiás (Morretes, 1952).

Comentários: Coletado um único exemplar bem preservado, em Corumbá (Corumbá de Goiás), no Estado de Goiás, pelo geólogo Dr. Armando Wohlers, a quem o autor dedicou o nome da espécie. A ocorrência da espécie, mencionada por Simone (2006) e Salvador et al. (2018b), em Corumbá, Estado do Mato Grosso do Sul, é um equivoco. 


\section{Gênero Mirinaba Morretes, 1952 Mirinaba antoninensis (Morretes, 1952)} Fig. 4D

Strophocheilus (Mirinaba) antoninensis Morretes, 1952: 113 (estampas 1-2, fig. 6); Morretes, 1953a: 63. Mirinaba antoninensis Leme, 1973: 329; Indrusiak \& Leme, 1985: 164 (figs. 1-2); Salgado \& Coelho, 2003: 156; Simone, 2006: 203 (fig. 765).

Distribuição: Antonina e Paranaguá, Estado do Paraná; São Vicente, Estado de São Paulo (Indrusiak \& Leme, 1985; Simone, 2006).

Comentários: Os exemplares da descrição original foram coletados pelo autor em agosto de 1948, na locali- dade chamada Fazenda Santa Olímpia, Cachoeira, município de Antonina, no Paraná.

\section{Mirinaba cadeadensis (Morretes, 1952) \\ Fig. 4E}

Strophocheilus (Mirinaba) cadeadensis Morretes, 1952: 115 (estampas 1-2, fig. 2); Morretes, 1953a: 63.

Mirinaba cadeadensis Leme, 1973: 329; Indrusiak \& Leme,

1985: 165 (figs. 3-4); Salgado \& Coelho, 2003: 156; Simone, 2006: 203 (fig. 766); Birckolz et al., 2013: 1561 (fig. 1).

Distribuição: Morretes, São José dos Pinhais, Paranaguá, Guaratuba, Matinhos, no Estado do Paraná (Birckolz et al., 2013).

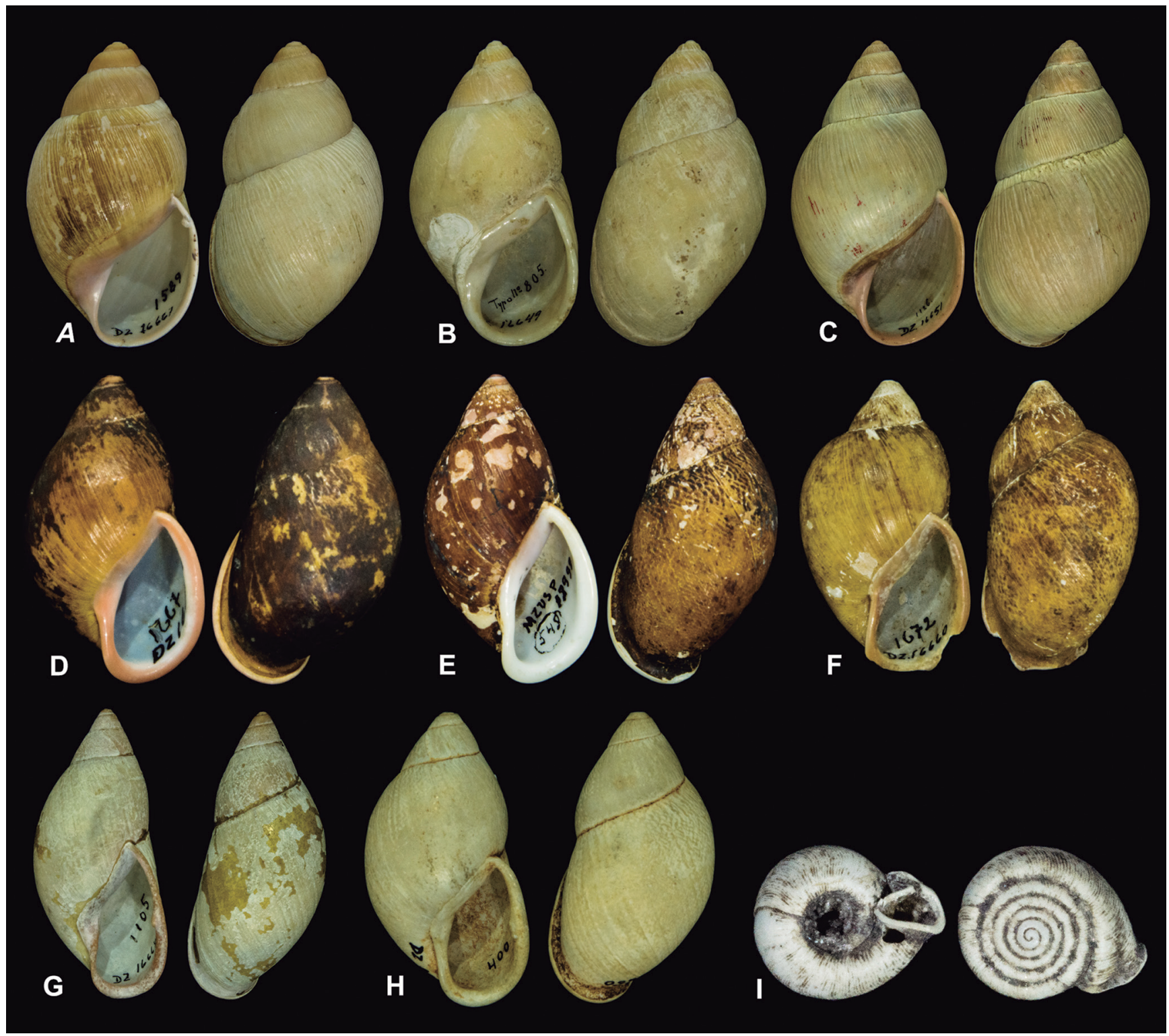

Figura 4. Espécies descritas por Lange de Morretes. H: altura da concha, L: largura maior da concha. (A) = Megalobulimus rolandianus, MZUSP 16667, Coleção Lange de Morretes, Holótipo, H=78,5 mm); (B) = Megalobulimus toriii, MZUSP 16649, Coleção Lange de Morretes, Holótipo, H = 75 mm; (C) = Megalobulimus wohlersi, MZUSP 16651, Coleção Lange de Morretes, Holótipo, H = 85 mm; (D) = Mirinaba antoninensis, MZUSP 16656, Coleção Lange de Morretes, Holótipo, H=41,5 mm; $(E)=$ Mirinaba cadeadensis, MZUSP 18998, Coleção Lange de Morretes, Holótipo, H=59 mm; (F) = Mirinaba curytibana, MZUSP 16660, Coleção Lange de Morretes, Holótipo, H= 45 mm; (G)=Mirinaba cuspidens, MZUSP 16664, Coleção Lange de Morretes, Holótipo, H=67,8 mm; (H) = Mirinaba jaussaudi, MZUSP 16652, Coleção Lange de Morretes, Holótipo, $\mathrm{H}=45,5 \mathrm{~mm} ;(\mathrm{I})=$ Entodina gionensis, MZUSP 54063, Coleção Ottorino de Fiore, Parátipo, H =4,4 mm. 
Comentários: As conchas da série-tipo foram coletadas em 28 de abril de 1935, por Ana Henkel, irmã de Frederico Lange de Morretes, e o marido dela, o renomado fotógrafo paranaense da estrada de ferro, Armin Henkel. A localidade-tipo é Nossa Senhora do Cadeado, região da Serra do Mar, no Paraná, junto à estrada de ferro Curitiba-Paranaguá.

\section{Mirinaba curytibana (Morretes, 1952)} Fig. 4F

Strophocheilus (Mirinaba) curitybanus Morretes, 1952: 115 (estampas 1-2, fig. 4); Morretes, 1953a: 63.

Mirinaba curytibana Leme, 1973: 329; Indrusiak \& Leme, 1985: 165 (figs. 5-6); Simone, 2006: 203 (fig. 767). Mirinaba curitybana Salgado \& Coelho, 2003: 156.

Distribuição: Pilarzinho (Curitiba) e Juruqui (atualmente município de Campo Magro), Estado do Paraná (Indrusiak \& Leme, 1985; Simone, 2006).

Comentários: Quatro exemplares foram coletados pelo autor em 1937, no bairro Pilarzinho, município de Curitiba, Estado do Paraná.

\section{Mirinaba cuspidens (Morretes, 1952) Fig. 4G}

Strophocheilus (Metara) cuspidens Morretes, 1952: 116 (estampas 1-2, fig. 5); Morretes, 1953a: 64.

Mirinaba cuspidens Leme, 1973: 329; Simone, 2006: 204 (fig. 768).

Distribuição: Brasil (Simone, 2006).

Comentários: Tanto a localidade-tipo, quanto o coletor desta espécie são duvidosas. As informações, segundo Lange de Morretes, são que sua procedência é Brasil, e o exemplar foi doado pelo seu amigo e biólogo, Dr. Hans Krüger, e originalmente pertencia à coleção do Museu "Olinda Schule", na cidade de São Paulo.

\section{Mirinaba jaussaudi (Morretes, 1937) Fig. 4H}

Coniclus jaussaudi Morretes, 1937a: 303 (estampa 2, figs. 1-5).

Strophocheilus planidens jaussaudi Bequaert, 1948: 41 (estampa 21, fig. 3).

Strophocheilus jaussaudi Morretes, 1949: 140.

Strophocheilus (Metara) jaussaudi Morretes, 1952: 112; Morretes, 1953a: 64.

Mirinaba jaussaudi Leme, 1973: 330; Salgado \& Coelho, 2003: 156; Simone, 2006: 204 (fig. 771); Birckolz \& Gernet, 2016: 1 (figs. 1-5).
Distribuição: Sengés, Iretama, Cornélio Procópio, Cerro Azul, Estado do Paraná; Apiaí, Estado de São Paulo (Morretes, 1954a; Birckolz \& Gernet, 2016).

\section{Superfamília Rhytidoidea Família Scolodontidae Gênero Entodina Ancey, 1887 Entodina gionensis Morretes, 1940 Fig. 41}

Entodina gionensis Morretes, 1940c: 257 (estampa 1, figs. 1-4); Morretes, 1949: 138; Simone, 2006: 223 (fig. 849); Salvador et al., 2016: 64 (figs. 20-22).

Distribuição: Sambaqui Vamiranga, Iguape, e Parque Estadual e Turístico Alto Ribeiro, no Estado de São Paulo; Estado de Santa Catarina (Salvador et al., 2016).

\section{CONSIDERAÇÕES FINAIS}

Atualmente, a maior parte da coleção particular de moluscos de Lange de Morretes encontra-se depositada no Museu de Zoologia da Universidade de São Paulo (Dornellas \& Simone, 2011; Cavallari et al., 2016). Outros espécimes malacológicos coletados por ele durante sua vida acadêmica estão depositados no Museu Nacional, Rio de Janeiro, no Museu de História Natural Capão da Imbuia e Museu Paranaense, ambos em Curitiba, na coleção do Laboratório de Ecologia Aplicada e Bioinvasões da UFPR e, possivelmente, também com particulares. No entanto, devido ao incêndio que ocorreu no Museu Nacional em 2 de setembro de 2018 (Zamudio et al., 2018), possivelmente, o material depositado naquele acervo foi destruído.

Lange de Morretes atuou como cientista em um período áureo para a ciência no Brasil, momento em que os museus ligados às ciências naturais existentes no país começam a se profissionalizar (Lopes, 2009). Entre esses museus destacam-se o Museu Paulista e o Museu Paranaense, locais aos quais Lange de Morretes esteve vinculado. O catálogo de moluscos por ele elaborado é o primeiro do país e, desde então, novas listas de espécies brasileiras são elaboradas tendo esse trabalho como base (Birckolz et al., 2016).

Após o falecimento de Lange de Morretes, em 1954, ocorreu um período de estagnação nas pesquisas malacológicas no Estado do Paraná (Colley, 2012; Colley et al., 2012), lacuna esta que se estendeu por cerca de 30 anos, tendo poucos trabalhos sido realizados na área neste período (e.g., Zanardini, 1965; Leme et al., 1979; Dutra \& Leme, 1985; Indrusiak \& Leme, 1985).

Pela importância e pioneirismo de seus trabalhos, é fundamental uma valorização histórica de sua obra, mostrando, para as novas gerações de pesquisadores, uma época pretérita, de dificuldades e paixões que está pouco a pouco desaparecendo no tempo. 


\section{AGRADECIMENTOS}

Agradecemos aos funcionários das bibliotecas do Museu de Zoologia da Universidade de São Paulo, do Museu Paranaense e do Museu de História Natural Capão da Imbuia, pela ajuda com a bibliografia. A Alexandre D. Pimenta, pelas informações sobre os lotes coletados por Lange de Morretes depositados no Museu Nacional.

\section{REFERÊNCIAS}

Agudo-Padrón, I.; Luz, J.S.; Lisboa, L.K. \& Zermiani, A.E. 2014. Additional twelve new records to inventory of continental mollusc species from Santa Catarina State, SC, Central Southern Brazil. Boletín de la Asociación Argentina de Malacología, 3(2): 11-20.

Bequaert, J.C. 1948. Monograph of the family Strophocheilidae, a Neotropical family of terrestrial molluscs. Bulletin of the Museum of Comparative Zoology, 100(1): 1-210.

Birckolz, C.J. \& Gernet, M.V. 2016. New record of Mirinaba jaussaudi (Gastropoda, Strophocheilidae) in Paraná state, southern Brazil, and rectification of a known locality. Strombus, 23(1-2): 1-5.

Birckolz, C.J.; Gernet, M.V. \& Serbena, A.L. 2013. Range extension of Mirinaba cadeadensis (Morretes, 1952) (Gastropoda:Pulmonata:Strophocheilidae) along the coast of Paraná, southern Brazil. Check List, 9(6): 1561-1563.

Birckolz, C.J.; Salvador, R.B.; Cavallari, D.C. \& Simone, L.R.L. 2016. Illustrated checklist of newly described (2006-2016) land and freshwater Gastropoda from Brazil. Archiv für Molluskenkunde, 145(2): 133-150.

Bouchet, P. \& Rocroi, J.-P. 2005. Classification and nomenclator of gastropod families. Malacologia, 47(1-2): 1-397.

Cavallari, D.C.; Simone, L.R.L.; Almeida, S.M.; Pedro, N.C. \& Carvalho, C.A. 2016. The mollusk collection of University of São Paulo Museum of Zoology: An overview and perspectives. Arquivos de Ciências do Mar, 49(supl.): $40-45$.

Claremont, M.; Vermeij, G.J.;Williams, S.T. \& Reid, D.G. 2013. Global phylogeny and new classification of the Rapaninae (Gastropoda: Muricidae), dominant molluscan predators on tropical rocky seashores. Molecular Phylogenetics and Evolution, 66(1): 91-102.

Colley, E. 2012. Moluscos terrestres e a malacologia paranaense: histórico e importância no cenário nacional. Estudos de Biologia, 34(82): 75-81.

Colley, E.; Simone, L.R.L. \& Loyola e Silva, J. 2012. Uma viagem pela história da Malacologia. Estudos de Biologia, 34(83): 175-190.

Corrêa, A.S. 2014. Alfredo Andersen: retratos e paisagens de um norueguês caboclo. São Paulo, Alameda Casa Editorial.

Dornellas, A.P.S. \& Simone, L.R.L. 2011. Annotated list of type specimens of mollusks deposited in Museu de Zoologia da Universidade de São Paulo, Brazil. Arquivos de Zoologia, 42(1): 1-81.

Dutra, A.V.C. \& Leme, J.L.M. 1985. Scutalus (Aposcutalus) atlanticus Subgênero e espécie novos (Gastropoda, Bulimulidae) do Brasil. Acta Biológica Paranaense, 14(1-4): 23-38.

Indrusiak, L.F. \& Leme, J.L.M. 1985. Anatomia comparada de três espécies de Mirinaba Morretes, 1952 (Gastropoda, Strophocheilidae) do Estado do Paraná, Brasil. Acta Biológica Paranaense, 14(1-4): 163-180.

Leme, J.L.M. 1973. Anatomy and systematics of the Neotropical Strophocheiloidea (Gastropoda, Pulmonata) with the description of a new family. Arquivos de Zoologia, 23(5): 295-337.

Leme, J.L.M.; Castro, R.L. \& Indrusiak L.F. 1979. Contribuição anatômica e histológica para o conhecimento de Mirinaba antoninensis (Morretes, 1952) (Gastropoda, Strophocheilidae). Papéis Avulsos de Zoologia, 32(14): 183-191.
Lopes, M.M. 2009. OBrasil descobre a pesquisa científica: Os museus e as ciências naturais no Século XIX. 2.ed. São Paulo, Editora Hucitec/Editora UnB.

Marini, A.C. 1988. Contribuição ao conhecimento de Thais mariae, Morretes, 1954 (Mollusca Prosobranchia). Revista Brasileira de Zoologia, 5(2): 311-323.

Morretes, F.L. [1931/1943]. Esperança. Texto manuscrito [local indefinido de depósito].

Morretes, F.L. 1937a. Dois novos gasteropodos pulmonados do Brasil. Revista do Museu Paulista, 23: 299-306.

Morretes, F.L. 1938a. Duas espécies novas de molluscos marinhos do Brasil. In: Silva, B. \& Travassos Filho, L. (Eds.). Livro Jubilar do Professor Lauro Travassos: editado para commemorar $025^{\circ}$ anniversario de suas actividades scientificas (1913-1938). Rio de Janeiro, Typographia do Instituto Oswaldo Cruz.

Morretes, F.L. 1940a. Algumas palavras sôbre novas ocorrências e maior distribuição de moluscos na costa do Brasil. Revista de Indústria Animal, São Paulo, 3(4): 184-187.

Morretes, F.L. 1940b. Novos Moluscos marinhos do Brasil. Arquivos de Zoologia do Estado de São Paulo, 2(7): 251-256.

Morretes, F.L. 1940c. Um novo Gasterópodo Pulmonado do Brasil. Arquivos de Zoologia do Estado de São Paulo, 2(8): 257-260.

Morretes, F.L. 1941. Rochanaia gutmansi, nov. gen. n. sp., novo Lamelibrânquio do Brasil. Revista Arquivos do Instituto Biológico, 12(5): 75-80.

Morretes, F.L. 1943. Contribuição ao Estudo da Fauna Brasileira de Moluscos: Resultados de uma pequena coleção de moluscos obtida pela excursão científica realizada pelo Instituto Osvaldo Cruz em outubro de 1938. Papéis Avulsos do Departamento de Zoologia, 3(7): 111-126.

Morretes, F.L. 1949. Ensaio de catálogo dos moluscos do Brasil. Arquivos do Museu Paranaense, 7: 5-216.

Morretes, F.L. 1952. Novas espécies brasileiras da família Strophocheilidae. Arquivos de Zoologia do Estado de São Paulo, 8(4): 109-126.

Morretes, F.L. 1953a. Adenda e corrigenda ao ensaio de catálogo dos moluscos do Brasil. Arquivos do Museu Paranaense, 10(1): 37-76.

Morretes, F.L. 1953b. 0 pinheiro na arte. Ilustração Brasileira, Rio de Janeiro, 44(224): 168-169, 274.

Morretes, F.L. 1953c. Relatório sobre as atividades de Frederico Lange de Morretes. In: Museu Paranaense. Livro de relatórios 1950-1969. Curitiba, Museu Paranaense.

Morretes, F.L. 1954a. Dois novos moluscos do Brasil. Arquivos do Museu Paranaense, 10(2): 331-336.

Morretes, F.L. 1954b. Nova Thais do Brasil. Arquivos do Museu Paranaense, 10(2): 339-340.

Morretes, F.L. 1954c. Sôbre Megalobulimus paranaguensis Pilsbry \& Ihering. Arquivos do Museu Paranaense, 10(2): 343-344.

Oliveira, M.P. \& Almeida, M.N. 1999. Conchas dos caramujos terrestres do Brasil. Juiz de Fora, MG, Editar.

Rios, E.C. 1970. Coastal Brazilian Seashells. Rio Grande, Fundação Cidade do Rio Grande/Museu Oceanográfico de Rio Grande.

Rios, E.C. 1975. Brazilian Marine Mollusks Iconography. Rio Grande, Fundação Universidade do Rio Grande.

Rios, E.C. 1985. Seashells of Brazil. Rio Grande, Fundação Universidade do Rio Grande.

Rios, E.C. 1994. Seashells of Brazil. 2.ed. Rio Grande, Fundação Universidade do Rio Grande.

Rios, E.C. 2009. Compendium of Brazilian Sea Shells. Rio Grande, Evangraf.

Rosenberg, G. 2009. Malacolog 4.1.1: A Database of Western Atlantic Marine Mollusca. Disponível em: http://www.malacolog.org. Acesso em: 17/04/2018.

Salgado, N.C. \& Coelho, A.C.S. 2003. Moluscos terrestres do Brasil (Gastrópodes operculados ou não, exclusive Veronicellidae, Milacidae e Limacidae). Revista de Biología Tropical, 51(supl. 3): 149-189. 
Salturi, L.A. 2007. Frederico Lange de Morretes, liberdade dentro de limites: trajetória do artista-cientista. (Dissertação de Mestrado). Curitiba, Universidade Federal do Paraná.

Salturi, L.A. 2009a. 0 caminho percorrido por Frederico Lange de Morretes (1892-1954). In:Szwako, J.E.L. \& Oliveira, M. (Eds.). Ensaios de sociologia e história intelectual do Paraná. Curitiba, Editora UFPR.

Salturi, L.A. 2009b. Paranismo, movimento artístico do sul do Brasil no início do século XX. Periferia, revista de recerca i formació em antropologia, Barcelona, 11: 1-22.

Salvador, R.B.; Cavallari, D.C. \& Simone, L.R.L. 2016. Taxonomical study on a sample of land snails from Alto Ribeira State Park (São Paulo, Brazil), with description of a new species. Archiv für Molluskenkunde, 145(1): 59-68.

Salvador, R.B.; Charles, L.; Simone, L.R.L. \& Maestrati, P. 2018a. Terrestrial gastropods from Pedra Talhada Biological Reserve, Alagoas state, Brazil, with description of a new species of Radiodiscus (Gastropoda: (haropidae). Archiv für Molluskenkunde, 147(1): 101-128.

Salvador, R.B.; Colley, E. \& Simone, L.R.L. 2018b. Terrestrial mollusks from the region of Corumbá and Maciço do Urucum, SW Brazil. Journal of Conchology, 43(1): 71-88.

Simone, L.R.L. 2003a. Relatório da Secção de Malacologia do Anno de 1936. Informativo SBMa, 34(146): 3-4.
Simone, L.R.L. 2003b. Relatório da Secção de Malacologia do Anno de 1937. Informativo SBMa, 34(146): 3-4.

Simone, L.R.L. 2006. Land and Freshwater Molluscs of Brazil. São Paulo, EGB/ FAPESP.

Simone, L.R.L. 2017. A new species of Thaisella (Neogastropoda: Muricidae) from Caribbean Guatemala, with accounts on the anatomy and taxonomy of the genus in the Western Atlantic. Archiv für Molluskenkunde, 146(1): $111-120$.

Zamudio, K.R.; Kellner, A.; Serejo, C.; Britto, M.R.; Castro, C.B.; Buckup, P.A.; Pires, D.O.; Couri, M.; Kury, A.B.; Cardoso, I.A.; Monné, M.L.; Pombal Jr., J.; Patiu, C.M.; Padula, V.; Pimenta, A.D.; Ventura, C.R.R.; Hajdu, E.; Zanol, J.; Bruna, E.M.; Fitzpatrick, J. \& Rocha, L.A. 2018. Lack of Science support fails Brazil. Science, 361(6409): 1322-1323.

Zanardini, F.I. 1960. Uma nova espécie de Agaronia do Paraná "Olividae, Mollusca". Boletim do Instituto de História Natural, Zoologia, Curitiba, 2: 1-3.

Zanardini, F.I. 1965. Nota sobre Diplodon e Anodontites (Mollusca-Pelecypoda) de rios de Curitiba (Paraná). Boletim do Instituto de História Natural, Zoologia, Curitiba, 6: 1-11. 